\title{
Monitoring asthma: no matter how?
}

\author{
Lara S. van den Wijngaart, Jolt Roukema and Peter J.F.M. Merkus
}

Affiliation: Radboud University Medical Centre, Amalia Children's Hospital, Dept of Paediatrics, Division of Respiratory Medicine and Allergy, Nijmegen, The Netherlands.

Correspondence: Lara S. van den Wijngaart, Radboud University Medical Centre, Amalia Children's Hospital, Dept of Paediatrics, Division of Respiratory Medicine and Allergy, Geert Grooteplein 10, Nijmegen, 6500 HB, The Netherlands. E-mail: Lara.vandenwijngaart@radboudumc.nl

The ultimate goal of asthma care is to obtain and maintain control, and optimise prognosis for patients [1]. Monitoring and treatment adjustments tailored to patients' needs and wishes are essential to achieve controlled asthma and are therefore a fundamental part of comprehensive asthma management. However, the best strategy for asthma monitoring and its frequency are still unknown [2]. In daily practice, healthcare professionals combine scheduled routine clinical visits, lung function tests, repeated targeted education and questionnaires to evaluate asthma control and/or quality of life. Furthermore, adherence to treatment is strongly associated with asthma control [1,3]. Although proper treatment of asthma could prevent exacerbations, several studies have suggested that the majority of patients do not have adequate control of their disease [4]. For a large part, this may be explained by suboptimal adherence; in patients with asthma adherence varies between 30 and 70\% [5-7].

For healthcare professionals it is often unclear whether the treatment is suboptimal or that medication dosage is adequate but the patient does not adhere to therapy. Reasons for medication nonadherence are numerous and complex, but one of the most common reasons for medication nonadherence is forgetfulness $[8,9]$. Several studies have been focusing on improving adherence to medication and remote monitoring of the use of inhalation medication by electronic devices (e.g. text messages, automated phone calls and audiovisual reminder devices) $[10,11]$.

The e-MATIC (e-Monitoring of Asthma Therapy to Improve Compliance in children) study by VASBINDER et al. [12] reported in this issue of the European Respiratory Journal is a randomised controlled trial aimed at improving adherence to inhaled corticosteroid (ICS) therapy using information technology. Real-time medication monitoring (RTMM) was used to record the time and date of administered ICS doses. The hypothesis was that RTMM would not only improve adherence, but also improve asthma control, asthma-related quality of life and reduce the number asthma exacerbations. Children with asthma $(n=209$, aged 4-11 years) were included and used RTMM. Participants were randomised to receive or not to receive SMS reminders when the prescribed dose of ICSs was at risk of omission.

The primary outcome was timing adherence to ICS, defined as the proportion of all prescribed doses that were inhaled within a 6 - $\mathrm{h}$ time frame around the planned inhalation (i.e. $3 \mathrm{~h}$ before to $3 \mathrm{~h}$ after), as recorded by the RTMM device. They found that children receiving SMS reminders had better timing adherence to ICS than children with RTMM alone, but observed no difference in asthma outcomes.

The important question is why, if adherence was better in the intervention group, was there no difference between groups in terms of asthma control, frequency of asthma exacerbations and asthma specific-quality of life? This may be due to several reasons.

First, measuring the time and date of administered ICS doses can only confirm that the medication was fired, but not whether this medication was actually inhaled. As the authors concluded, this may result in overestimation of actual adherence to ICS monitored by RTMM. Secondly, patients in both groups may

Received: June 202016 | Accepted: June 222016

Conflict of interest: Disclosures can be found alongside this article at erj.ersjournals.com

Copyright @ERS 2016 
have been more adherent than they would have been in daily practice, as they were aware that they were being observed. Thirdly, only selected patients that were motivated to improve their adherence participated in the study.

In addition, registered doses may not have been administered adequately due to poor inhalation technique, which is known to have a substantial effect on the efficacy of ICS. It is also possible that the improvement in medication adherence was not large enough to result in improved clinical asthma outcomes at an individual patient level. All patients have their own threshold/balance between ICS dosage and asthma control. It is conceivable that, although $50 \%$ of the medication was forgotten and SMS reminders were received, patients balanced this out themselves. Another explanation for the fact that improved adherence was not accompanied by lower asthma-related care utilisation could be that the patients were already well-controlled at inclusion, leaving little room for relevant improvement. The small number of events and the high childhood asthma control test score at the start of the study probably reflect the fairly well-controlled asthma of the participants, suggesting the need for a study in less well-controlled patients.

Nevertheless, the results of VASBINDER et al. [12] are consistent with earlier published studies focusing on reminder systems to improve adherence to asthma medication [10]. None of these studies were able to document a change in clinical asthma outcomes or asthma-related quality of life.

But how could interventions such as RTMM plus SMS reminders be applied in such a way that asthma control is improved? VASBINDER et al. [12] only focused on adherence in young children, aged 4-11 years. But poor adherence, and risk for severe asthma attacks, may be a much greater issue in adolescents. Treating teenagers with asthma is a challenge because of several factors such as poor adherence, denial of disease and underreporting of symptoms [13].

So it could very well be that interventions such as RTMM plus SMS reminders may be much more, or only, effective for selected patients (e.g. adolescents, patients with severe asthma and poor perceivers) and future research should focus on the use of RTMM in these subgroups.

Another interesting question is how sustainability of the effect can be guaranteed. New devices like RTMM are interesting, especially for children, but their interest in such devices will diminish over time. Besides achieving an improvement in self-management, the biggest challenge for healthcare professionals is to maintain the effectuated changes in self-management. Regrettably, a solution to this challenge cannot be given yet, but we are convinced that positive incentives will help in this group.

In an ideal world, monitoring asthma in children is tailored to every individual patient. This so-called "personalised asthma care" uses specific monitoring tools for different patients, preferably using different domains. As healthcare professionals we should stimulate awareness and self-management, and through that enhance adherence to treatment. Especially in adolescents we should emphasise shared decision making and find ways to communicate effectively.

To date, monitoring asthma is essential and as a healthcare professional one might think that it may not matter how you do this, as long as you do. We think that every monitoring strategy may work as an intervention, which may already and finally lead to increased awareness, better adherence and better asthma control.

The use of novel information technologies in monitoring asthma is inevitable and also very attractive because eHealth interventions provide great opportunities to monitor patients remotely and facilitate communication with the child or the parents. In subgroups of patients, eHealth monitoring using monthly questionnaires may serve as a partial replacement for face-to-face outpatient visits, thus facilitating a reduction in the number of outpatient visits. Other children may be stimulated by personalised feedback on their asthma scores or adherence and/or have access to online charts summarising remote monitoring of their ICS use. Some patients will only require an alert when their device is almost empty. The concept of a virtual asthma clinic, with an individualised monitoring strategy is an attractive and very realistic option in current asthma management. Such a clinic can be incorporated into electronic patient files, and can be accessible for asthmatic children so they can communicate easily with their asthma team, find reliable information about their disease and download their individual treatment plan or action plan in case of an asthma exacerbation.

Introducing personalised e-Health as part of current asthma management will play a valuable role in developing and reinforcing better adherence and asthma monitoring in general. Developments such as RTMM with SMS notifications may not have added value in all, but may be exactly the thing needed in subgroups of (adolescent?) patients with asthma. In the world of e-Health tools to enhance adherence and asthma control there is probably no "one size fits all". We all agree it is important that we monitor, but the how may be crucial. Similar to the way in which as asthma treatment should be personalised, the chosen monitoring tools should also fit the patient. 


\section{References}

1 Global Initiative for Asthma (GINA). Global Strategy for Asthma Management and Prevention, updated 2011. Available from: http://ginasthma.org/

2 Lødrup Carlsen KC, Pijnenburg MW, ERS Task Force Monitoring Asthma in Children. Monitoring asthma in childhood. Eur Respir Rev 2015; 24: 178-186.

3 Rottier BL, Eber E, Hedlin G, et al. Monitoring asthma in childhood: management-related issues. Eur Respir Rev 2015; 24: 194-203.

4 Chapman KR, Boulet LP, Rea RM, et al. Suboptimal asthma control: prevalence, detection and consequences in general practice. Eur Respir J 2008; 31: 320-325.

5 Klok T, Kaptein AA, Brand PL. Non-adherence in children with asthma reviewed: The need for improvement of asthma care and medical education. Pediatr Allergy Immunol 2015; 26: 197-205.

6 Morton RW, Everard ML, Elphick HE. Adherence in childhood asthma: the elephant in the room. Arch Dis Child 2014; 99: 949-953.

7 de Groot EP, Kreggemeijer WJ, Brand PL. Getting the basics right resolves most cases of uncontrolled and problematic asthma. Acta Paediatr 2015; 104: 916-921.

8 Weinstein AG. Should patients with persistent severe asthma be monitored for medication adherence? Ann Allergy Asthma Immunol 2005; 94: 251-257.

9 Burgess SW, Sly PD, Morawska A, et al. Assessing adherence and factors associated with adherence in young children with asthma. Respirology 2008; 13: 559-563.

10 Tran N, Coffman JM, Sumino K, et al. Patient reminder systems and asthma medication adherence: a systematic review. J Asthma 2014; 51: 536-543.

11 Ingerski LM, Hente EA, Modi AC, et al. Electronic measurement of medication adherence in pediatric chronic illness: a review of measures. J Pediatr 2011; 159: 528-534.

12 Vasbinder EC, Goossens LMA, Rutten-van Mölken MPMH, et al. e-Monitoring of Asthma Therapy to Improve Compliance in children (e-MATIC): a randomised controlled trial. Eur Respir J 2016; 48: 758-767.

13 Sadof M, Kaslovsky R. Adolescent asthma: a developmental approach. Curr Opin Pediatr 2011; 23: 758-767. 